\title{
HAIRY ROOT CULTURE OF AN IMPORTANT MEDICINAL PLANT: COLEUS FORSKOHLII
}

\author{
UMA MAHESWARI R. ${ }^{*}$, SELVAMURUGAN C. ${ }^{2}$, JAYABARATH J. ${ }^{3}$, LAKSHMI PRABHA A. ${ }^{4}$ \\ 1,4Department of Plant Science, Bharathidasan University, Trichy, 24, India \\ 2,3Head, Department of Biotechnology, Pavendar Bharathidasan College of Engineering \& Technology, Trichy, 24, India \\ *Corresponding Author: Email - umakseven@gmail.com , barath_bio@yahoo.co.in
}

Received: June 19, 2011; Accepted: July 19, 2011

\begin{abstract}
An experiment was conducted for induction \& establishment of Agrobacterium mediated hairy root culture of Coleus forskohlii was developed \& co-cultivation on hormone free semisolid MS Medium with B5vitamins.Sterilization period was standardized for the stem \& leaf explant using $0.1 \%$ bavistin and $0.1 \% \mathrm{Hgcl}_{2}$ with varied intervals It resulted in the emergence of hairy roots from the leaf explant, stem explant after $20^{\text {th }}$ day of infection .The transformation of hairy roots was established and confirmation of forskolin content was done by HPLC. Selection of high forskolin can provide an alternative source of large scale production of forskolin.
\end{abstract}

Keywords- Agrobacterium rhizogene, Coleus forskohlii ,Hairy root, HPLC

\begin{abstract}
Introduction
Coleus forskohlii is a botanical that has been used since ancient times in Hindu and Ayurvedic traditional medicine. The root portion of the plant has been traditionally used for medicinal purposes and contains the active constituent, forskolin. Forskolin was named after the Finnish botanist, Forska Forskolin content has been found to vary from 0.07 to $0.59 \%$ of dry tubers and just $1 \mathrm{~g}$ of forskolin costs $\$ 85$, showing the importance of this crop (Gowda 2000). This alkaloid has the unique property of activating all hormone-sensitive adenylate cyclase enzymes in biological systems (De souza \& Shah 1988). Because of continuous collection of tubers from its wild sources, this plant has been included in the list of endangered species. Recently farmers have started to row it as a crop because of its economic potential (Vishwakarma et al. 1988). Coleus forskohlii is a perennial member of the mint (Lamiaceae) family and grows in the subtropical temperate climates of India, Nepal, Sri Lanka, and Thailand. Plant height is approximately 1-2 feet and its striking leaves are teardrop shaped, shimmering green framing a bright purple center; leaf color varies depending on the amount of shade. A cluster of stalked pale purple or blue flowers branches off a single stem. The root stock is typically golden brown, thick, fibrous, and radially spreading. The diterpene forskolin, derived from the root of the plant, is the primary constituent of clinical interest in Coleus forskohlii. It was discovered by Western scientists in 1974 and was initially referred to as coleonol. Since that time, as other coleonols and diterpenoids have been identified, the name was changed to forskolin. Forskolin is responsible for virtually all pharmacological activities attributed to Coleus forskohlii; extracts of this constituent have been used in nearly all existing studies. There is
\end{abstract}

evidence, however, that other plant constituents, such as volatile oils and other diterpenoids and coleonols, may contribute to the pharmacological activity and absorption of forskolin. Detailed analysis reveals approximately 20 constituents in various parts of the plant, but forskolin and other coleonols are present only in the root portion. Infection of plant tissues with Rhizobium rhizogenes, also known as Agobacterium rhizogenes (young et al. 2001),results in massive proliferation of adventitious roots from the inoculation site. These differentiated tissues are stably transformed by the Ri (root inducing) plasmid from R.rhizogenes and may be propagated indefinitely on hormone free media. Plant roots are an especially rich source of secondary metabolites such as terpenoids, alkaloids, and isoflavanoids. Hairy root cultures have been induced in many plant species leading to the invitro production of numerous plant secondary metabolites and pharmacologically active compounds (Brigham et al. 1999; Flores et al. 1999; Giri and Narasu 2000; Saito et al. 2001; Rao and Ravishankar 2002; Souret et al. 2003; Lorence et al. 2004). Although cell suspension cultures often lose the ability to synthesize secondary products after repeated serial transfers, hairy root cultures are usually quite stable in their biosynthetic capacity. Another benefit of hairy root cultures is that they are easily grown from small-scale shake cultures to bioreactors suitable for larger scale production of the compounds of interest (Palazón et al. 2003. Given the potential benefits of forskolin for biomedical and other applications, we sought a method to produce this forskolin and related compounds from cultures. Here, we report conditions for establishing hairy root cultures from coleus forskohlii and show that high levels of forskolin and related compounds are present in hairy root tissues of Coleus forskohlii. 


\section{Materials and Method Plant material}

Plant material was collected from the Department of Plant Biotechnology, University of agricultural sciences, GKVK, Bangalore-560065, India. Various medicinal plants like Cathyranthus roseus, Roulfia serpentine, Withania somnifera were used. Among them, Coleus forskohlii gave good results. So, further work carried on with Coleus forskohlii. Leaf explants of approximately 0.25 to $1.25 \mathrm{~cm}^{2}$ and stem explants ranging from 0.55 to $1.50 \mathrm{cms}$ with damaged main vein were incubated for 20 min in $20 \mathrm{ml}$ of liquid MS medium containing $100 \mathrm{ml}$ of a bacterial culture grown overnight, and were then transferred to solid MS medium for another 2 days. Assessment of optimum sterilization period of chemical sterilant to obtain effective surface sterilization of stems/leaves to produce maximum roots transformation was done. Average root number/root length per explant were recorded. The cloned roots were maintained in the antibiotic treated medium.

\section{Transformation by Agrobacterium rhizogenes}

Different strains of Agrobacterium rhizogenes such as A4, ATCC18534, MTCC533 were obtained from different places \& standardized a suitable growth medium for A.rhizogene strain Transformation Coleus forskohlii with Ri-plasmid of $A$. rhizogenes for root organ culture to improve production of secondary metabolites. The sterile Leaf \& stem explants of Coleus forskohlii plants were cut and injured. Then exised explants used for transformation by Agrobacterium rhizogenes ATCC 15834 was standardized. Isolation and structure determination of RA in C. forskohlii hairy roots were induced by infection of Agrobacterium rhizogenes MAFF 03-01724 (Sasaki et al., 1998).

\section{Hairy root culture}

Hairy roots were cultured at 21/18 degree celcius in 16/8 $\mathrm{hrs}$ day/night regime, in the dark. Five $1-\mathrm{cm}$ tips of roots grown on solid media were placed in 250-ml Erlenmeyer flasks, and then well established roots were maintained on Basal M.S media in petriplates and transferred to fermentor when required for large scale production. Thereafter, explants were transferred to cefotaximecontaining (250mgml21) medium to eliminate the bacteria. One-cm tips of roots that grew on the explants were excised and placed on MS medium containing cefotaxime $(250 \mathrm{mgml} 21)$ in petridishes where they grew and branched further. After some transfers, the growth of roots was gradually stabilized, in this way the hairy root culture was established for detection of forskolin

\section{Identification of forskolin by HPLC}

Identification of coumpound was done by High Pressure Liquid Chromatography. Quantity of forskolin $\mathrm{mg} / \mathrm{g}$ were calculated. Forskolin was identified in transformed roots by HPLC . HPLC emerged as an efficient method of choice for analytical puposes, the sample required is also very low for this technique and forskolin sample will be analysed satisfactorily. As a result of transformation the increased amount of forskolin in a transformants was confirmed by HPLC.

\section{Results \& Discussion}

Agrobacterium rhizogene and the specified media were used for transformation work was standardized with Carrot Disk Method. The virulence of the strain was tested by Carrot Disk Method(Fig1).Among these four strains,ATCC15834 has given good results for induction of hairy roots.(Table1) and was maintained on YEMA medium. A similar observation for production of Rosmarinic acid was also made from experiments on treatment in suspension cultures of C. blumei (Szabo et al., 1999) and L. erythrorhizon (Mizukami et al., 1993) with YE and MJA. Forskolin production in in vitro cultures of $C$. forskohlii has been reported by Mersinger et al. (1988), Krombholz et al. (1992) and Sen et al. (1992), and the maximum forskolin productivity was $0.073 \%$, $0.1 \%$ and $0.01 \%$, respectively. In contrast, the forskolin content of hairy root (B9) induced by $A$. rhizogenes MAFF 03-01724 was found to be high compare to other species .Similarly, hairy root cultures of $C$. forskohlii in three different media were cultured on WP, MS and B5 (Gamborg et al. 1968) solid media containing 3\% sucrose in the dark for 1-2 weeks.

\section{Hairy Root Culture}

Hairy root cultures were initiated from the leaf \& stem explants of coleus foskohlii after 20 days of incubation with Agrobacterium rhizogeneATCC15834. Compared to non-treated control plants, this indicated that the tansformation efficiency was $100 \%$.Figure 2 Morphology of hairy roots induction on leaf \& stem explant after $20^{\text {th }}$ day of incubation. Roots were variable in their morphology immediately after induction. Some roots were thick in diameter and brown (A) or white (B). Other morphologies included very thin roots $(C)$ and highly branched roots (D). Generally, hairy root cultures grow rapidly on media without any exogenous growth regulators and are genetically and biochemically stable, and for these reasons are promising systems for producing secondary metabolites $C$. forskohlii hairy root clones B8 and B9 were cultured in hormone-free MS, B5 and WP liquid media in the dark. Both clones grew well in WP medium (Fig. 2). By HPLC analysis, however, more forskolin was detected in clone B9 cultured in WP medium (Fig. 3)

\section{Effect of $0.1 \%$ Bavistin And $0.1 \% \mathrm{Hgcl}_{2}$ On stem \& Leaf Explants}

Sterilization was standardized for stem \& leaf explant using $0.1 \%$ bavistin and $0.15 \mathrm{Hgcl}_{2}$ with varied time intervals (Table2).Significant differences were obtained among sterilization period combination with respect to percent explant survival ranging from 5 to 100 percent for stem \& 5 to $95 \%$ for leaf explants per replication.10.0+6.0\&10.0+8.0 minutes of bavistin and $\mathrm{HgCl}_{2}$ were found to be higher than that of all treatments. 
Screening of leaf size and stem length for root induction on $20^{\text {th }}$ day of incubation

Selection of optimum size of the explant is important for transformation. The experiment was conducted with leaf area ranging from 0.25 to $1.25 \mathrm{sq} \mathrm{cms}$ and stem length ranging from 0.55 to $1,50 \mathrm{cms}$ (table3)

2.4 Average Root Length Obtained from the treatment: Treatment differed significantly with respect to average root length per explant at various bacterial cell densities on $10^{\text {th }}$ and $20^{\text {th }}$ cultivation. On the $10^{\text {th }}$ day of cocultivation observation were recorded (TABLE4).Treatments ranging from 0 to $3.98 \mathrm{~cm}$ of root length per leaf explant and 0 to $2.98 \mathrm{cms}$ root length per stem .The treatment with $1.25 \mathrm{sq} . \mathrm{cm}$, leaf explants produced significantly higher average root length (3.98 $\mathrm{cm})$ (Fig4). The rest of the treatments were intermediate.

\section{Maintenance of Transformed Root Culture}

Well established roots were maintained on Basal media in petriplate and transferred to fermentor when required for large scale production.(Fig5) $C$. forskohlii hairy root cultures gave a main product which was determined to be Forskolin (Figure I) HPLC Analysis of Forskolin: Quantification of forskolin was done by the following method described earlier (Mukherjee et al.1996). HPLC analysis was performed on a C-18 ODS * $4.5 \mathrm{~mm}$ coloumn (flow rate: $1.2 \mathrm{ml} / \mathrm{min}$ under absorbance 0.005 using Methanol $\quad$ Water $7: 1$ ratio H3PO4/60:40V/V.forskolin was detected 210nm and its retention time compared with that of standard forskolin (stigma) Forskolin production in in vitro cultures of $C$. forskohlii has been reported by Mersinger et al. (1988), Krombholz et al. (1992) and Sen et al. (1992), and the maximum forskolin productivity was $0.073 \%, 0.1 \%$ and $0.01 \%$, respectively. In contrast, the forskolin content of hairy root (B9) induced by $A$. rhizogenes MAFF 0301724 was high (Krombholz et al. (1992) higher than has been reported previously and was compare- 458 These results are similar to those HPLC elution of extracts of hairy roots showed the presence of forskolin, with a retention time of 15.70 min has been reported by Wei hi \& Yoshihasa Asada et al . (2004). Similarly, there has been reported earlier for increase in concentration of forskolin of Coleus forskhlii because of inoculation with micro-organism (G.mosseae + T.viride) (X.U.Boby and D.J. Bagyaraj),2002. A similar trend was also noticed in hairy roots of maize by Agrobacterium rhizogene showed vigorous growth in hormone free media (X.U.Hongwei, ZHOUxiaofu, Lu jingmei,WANG Xing Zhi,2005). Auxin free basal medium is efficient for root induction was also been reported by Monier and Oehatt

In this present study, identification \& quantification of Forskolin in A. rhizogene ATCC15834 transformed hairy roots of coleus forskohlii was done by HPLC.(Fig6).. The amount of forskolin in hairy root of coleus forskohlii determined here is promising

\section{Conclusion}

The present communication presents an efficient protocol for the successful production of $A$. rhizogene ATCC15834 transformed hairy root culture in Coleus forskohlii Biomass of hairy roots increases quickly in culture so it would be reasonable to expect hairy root culture of Coleus forskohlii would be promising for large scale production of Forskolin.

\section{References}

[1] Abraham Z., Srivastava A.K. and Bagchi C.D. Current Science, 57:1337-1339.

[2] Alternative Medicine Review u Volume 11, Number $1 \mathrm{u}$ 2006 Page 47

[3] Binns A.N. and Thomashow M.F. (1998) Annu.Rev.Microbiol.,45; 575-606.

[4] Brigham L.A., Michaels P.J., Flores H.E. (1999) Plant Physiol. 119: 417-428.

[5] Ho H. J., Farrand S.K., Noel G.R., Widholm J.M. (2000) Planta 210: 195-204.

[6] Forskohl's Coleus - Coleus forskohlii BRIQ (Lamiaceae) Phytochemicals. chromadex.com/Phytosearch/Forskolin.htm [2006]

[7] Flores H.E., Vivanco J. M., Loyola-Vargas L. M. '(1999) Trends Plant Sci. 4: 220-226.

[8] Giri A., Narasu M. L. (2001) Plant Physiol. 127: 13611366.

[9] Gamborg O.L., Miller R.A., Ojima K. (1968) Exp Cell Res 50:151-158.

[10] Gowda S. (2000) Agro India 8,27-28.

[11] Lorence A., Medina-Bolivar F., Nessler C. L. (2004) Plant Cell Rep. 22: 437-441.

[12] Mano Y., Ohkawa H., Yamada Y. (1989) Plant Sci. 59: 191-201.

[13] Matlin S. A., Zhou R. H., Games D. E., Jones A., Ramsey E. D. (1984) J. High Resolut. Chromatogr. Chromatogr. Common.7: 196-202.

[14] Metzger H., Lindner E. (1981) Arzneimittelforschung, 31:1248-1250.

[15] Murashige T., Skoog F. (1962) Physiol. Plant. 15: 473497. 
Standardization of Agrobacterium rhizogenes strains

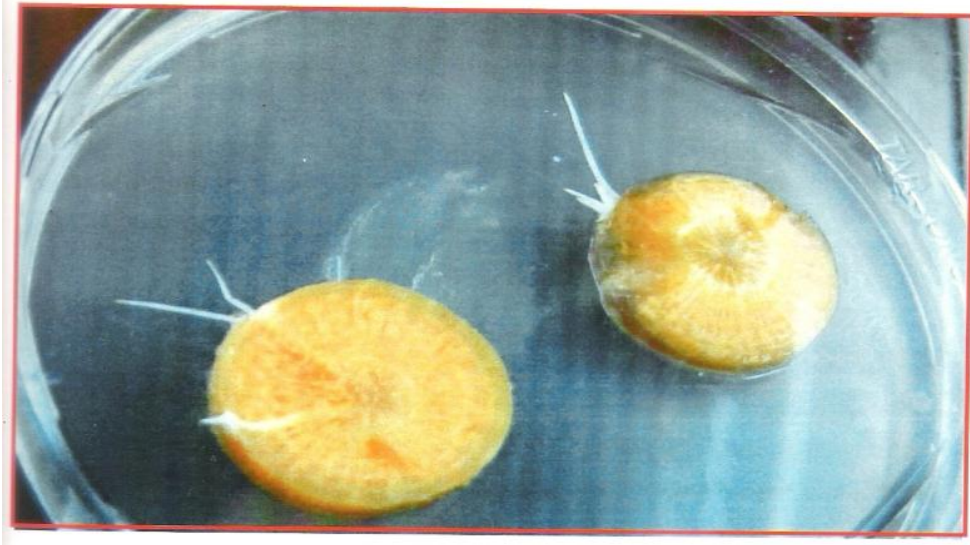

Fig. 1

Plate Showing Carrot Disc Method

- . -

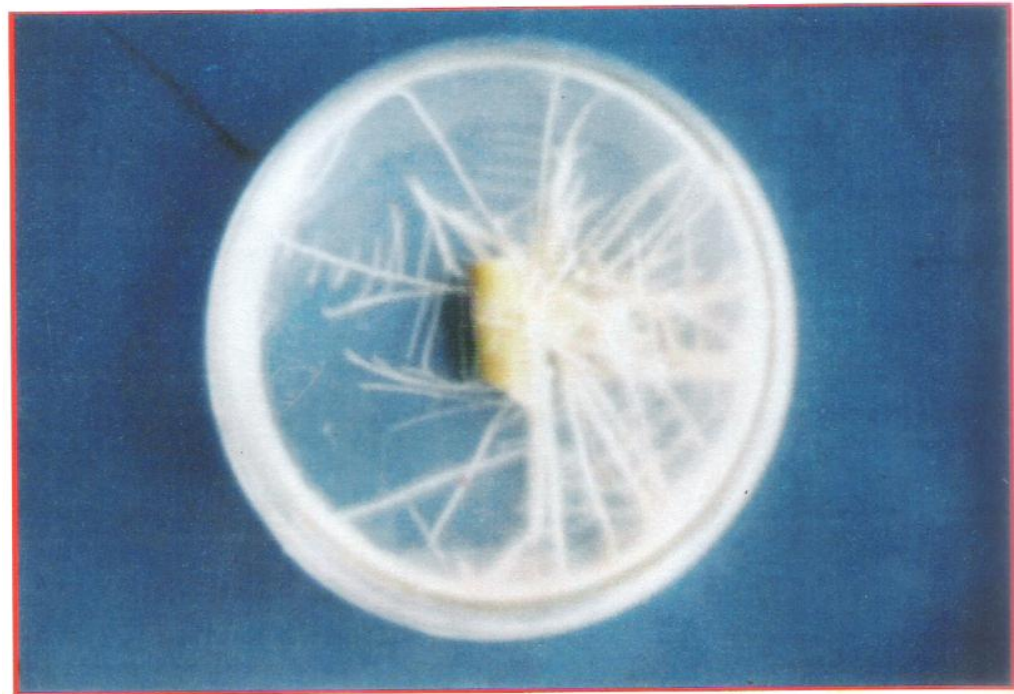

fig-2

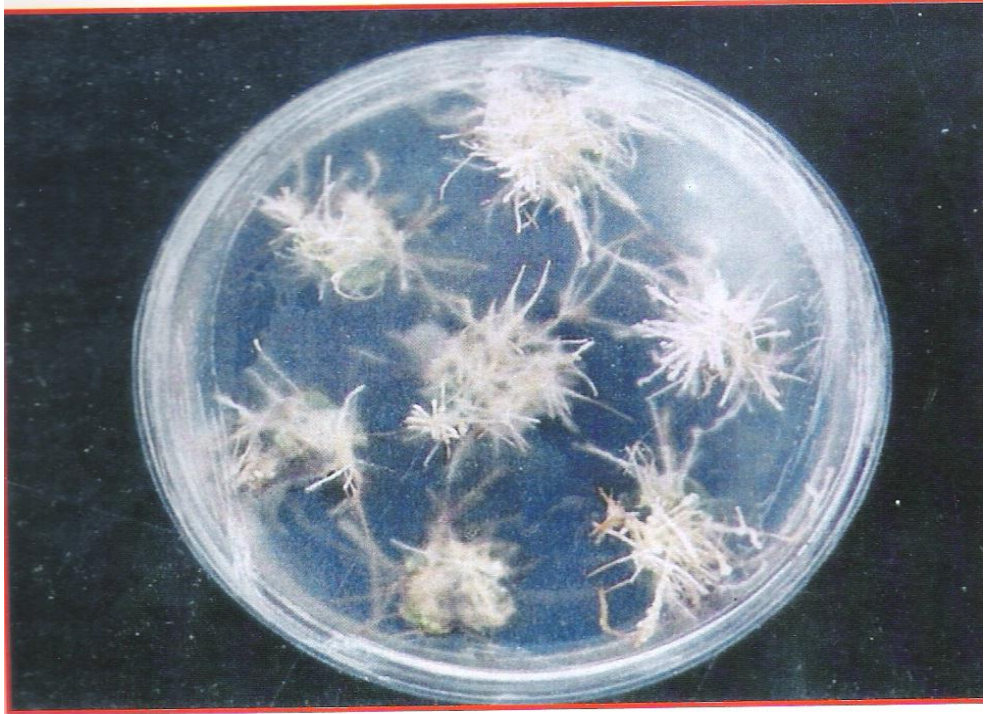

Fi@. 3A 


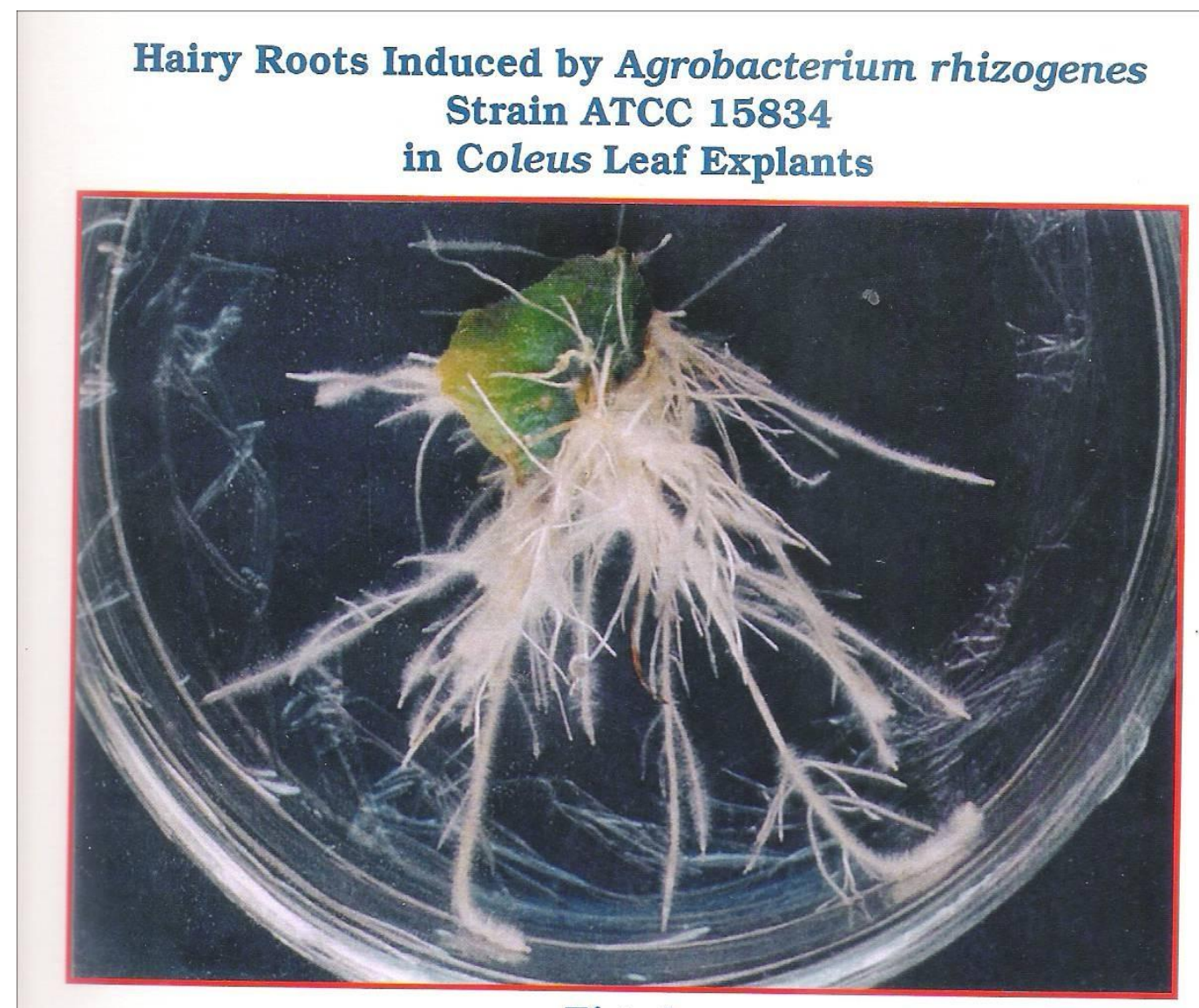

Fig. 4

(i) Normal View

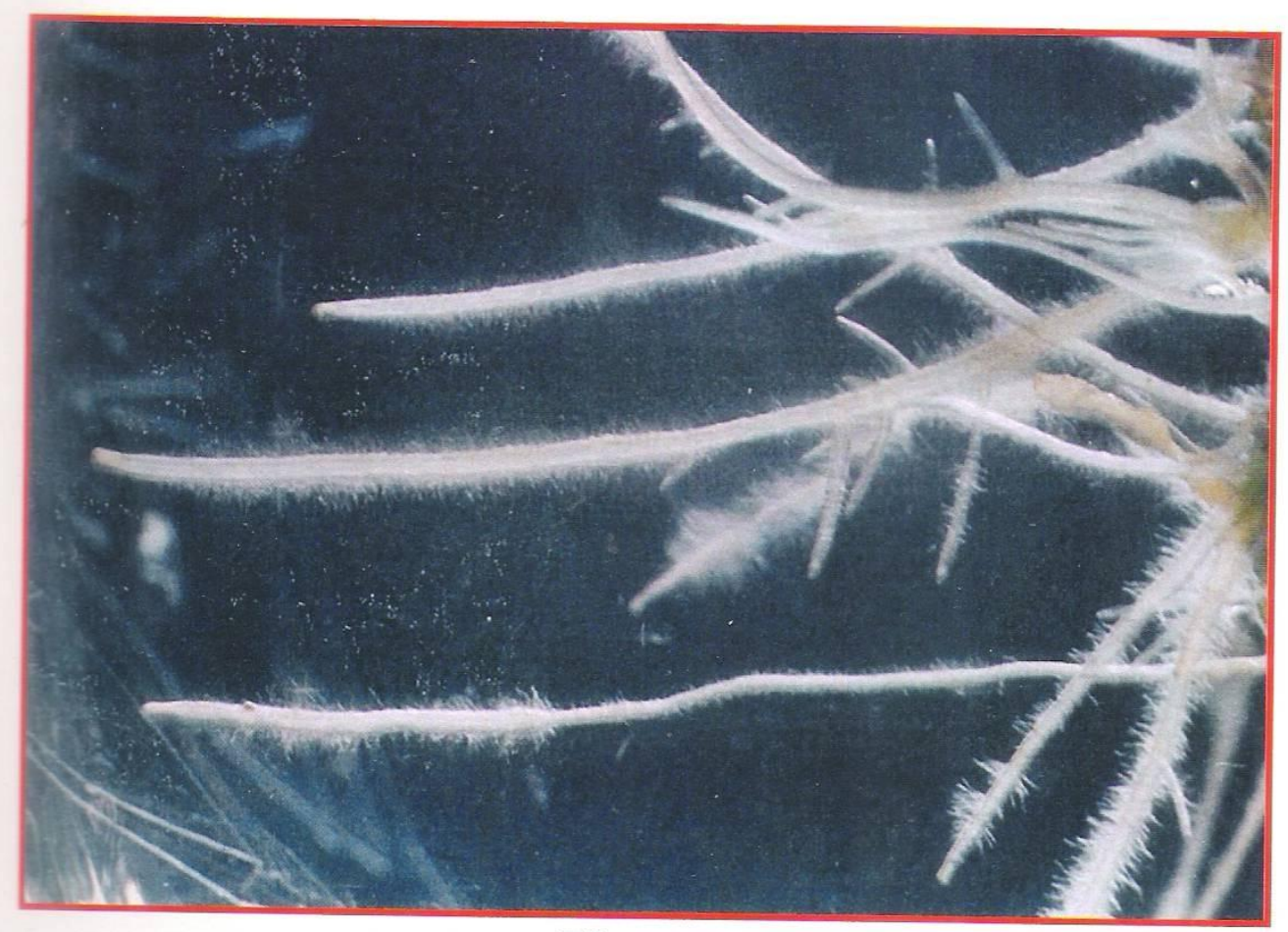

Fig. 4A

(ii) Closer View 


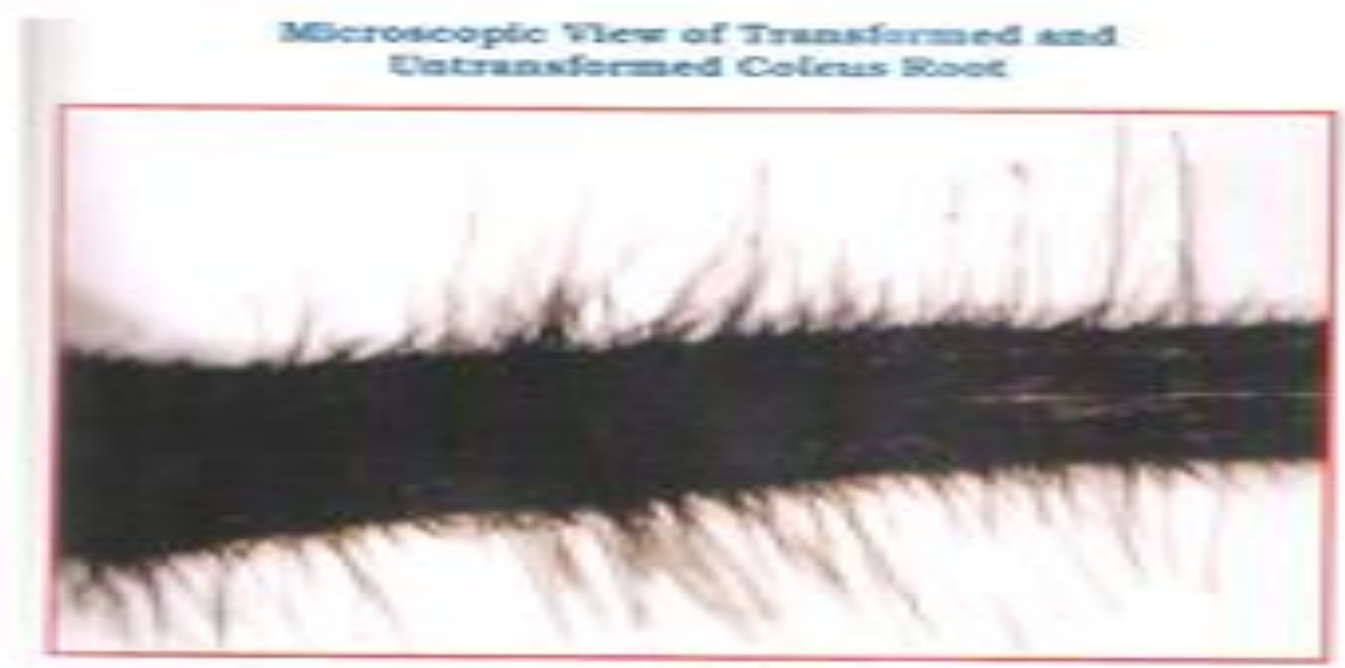

Iys. 5

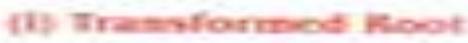

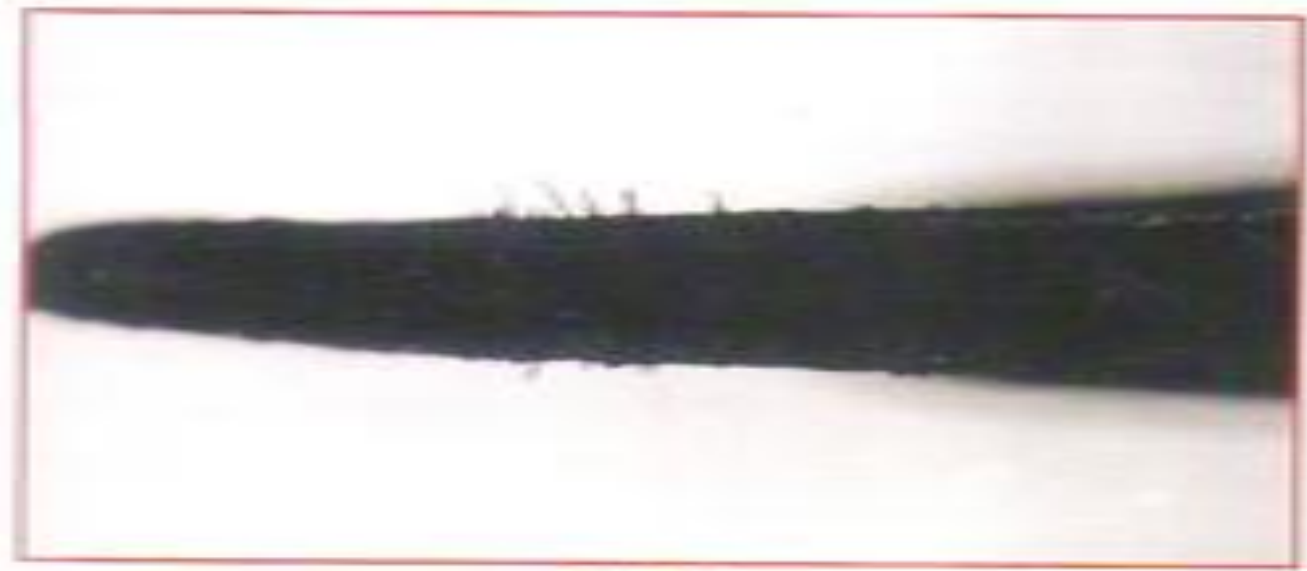

Fls: sas

Ean Entramsfourmed Rocet.

DAD1 C, Sh=210,8 Ref $=360,100$ (FORSK130080007.D)

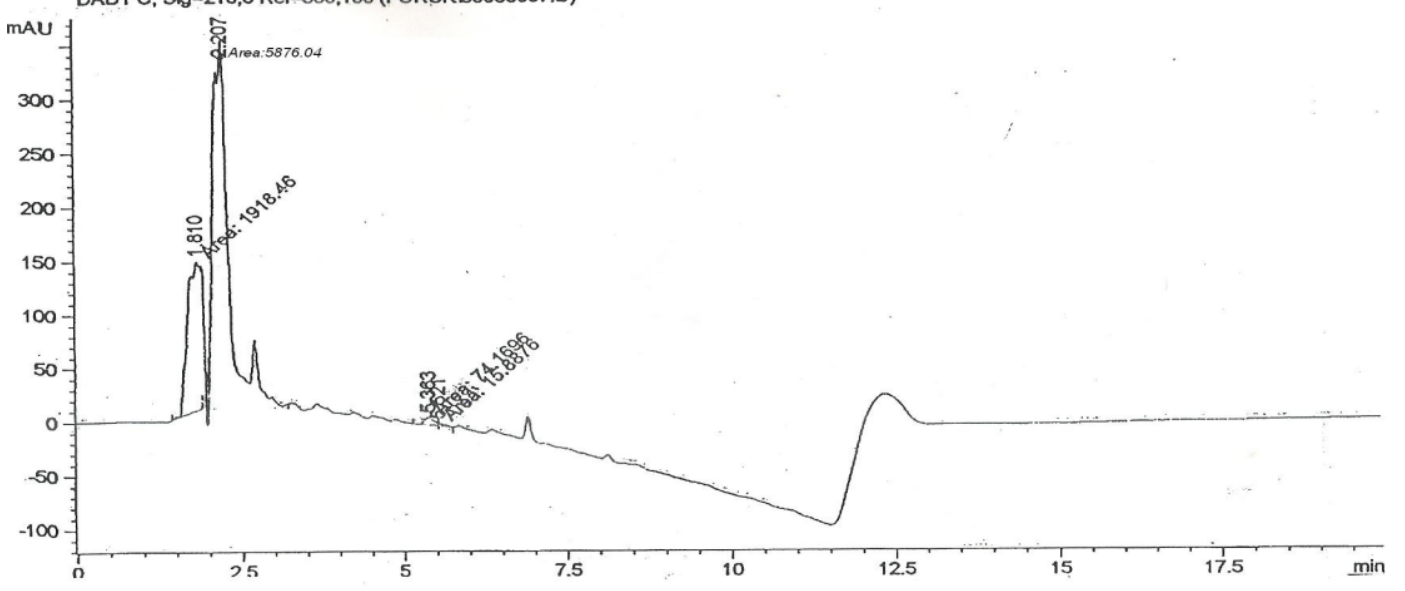


Hairy root culture of an important medicinal plant : Coleus forskohlii

Table 1-Strains were collected from different sources as given bellow:

\begin{tabular}{|c|l|c|}
\hline STRAINS & SOURCE & MEDIUM \\
\hline A4 & NCL,Pune & NA \\
\hline MTCC533 & CiMAP,Lucrow, Washington USA & YEMA \\
\hline A4 & Madurai Kamaraj University,Madurai & YEP \\
\hline ATCC15834 & Leiden University Nether lands & YEMA \\
\hline
\end{tabular}

Table 2- Effect of $0.1 \%$ Bavistin And $0.1 \% \mathrm{Hgcl}_{2}$ On stem \& Leaf explants

\begin{tabular}{|l|r|r|r|r|}
\hline $\begin{array}{l}\text { Sterilization period } \\
\text { (Minutes)Bavitin }+ \\
\mathrm{Hgcl} 2\end{array}$ & $\begin{array}{l}\text { Explant survival } \\
\text { Stem Number }\end{array}$ & \%Response & $\begin{array}{l}\text { Explant survival } \\
\text { Leaf Number }\end{array}$ & \%Response \\
\hline $00.0+0.0$ & 0.25 & 5 & 0.25 & 5 \\
\hline $10.0+2.0$ & 0.5 & 30 & 1.25 & 25 \\
\hline $10.0+4.0$ & 2.5 & 60 & 2.25 & 45 \\
\hline $10.0+6.0$ & 5.5 & 100 & 4.75 & 95 \\
\hline $10.0+8.0$ & 4.5 & 90 & 4.25 & 85 \\
\hline $10.10+10.0$ & 4 & 80 & 3.5 & 70 \\
\hline Mean & 2.79 & 60.8 & 2.7 & 54 \\
\hline
\end{tabular}

Effect of $0.1 \%$ Bavistin And $0.1 \% \mathrm{Hgcl}_{2}$ On stem \& Leaf explants

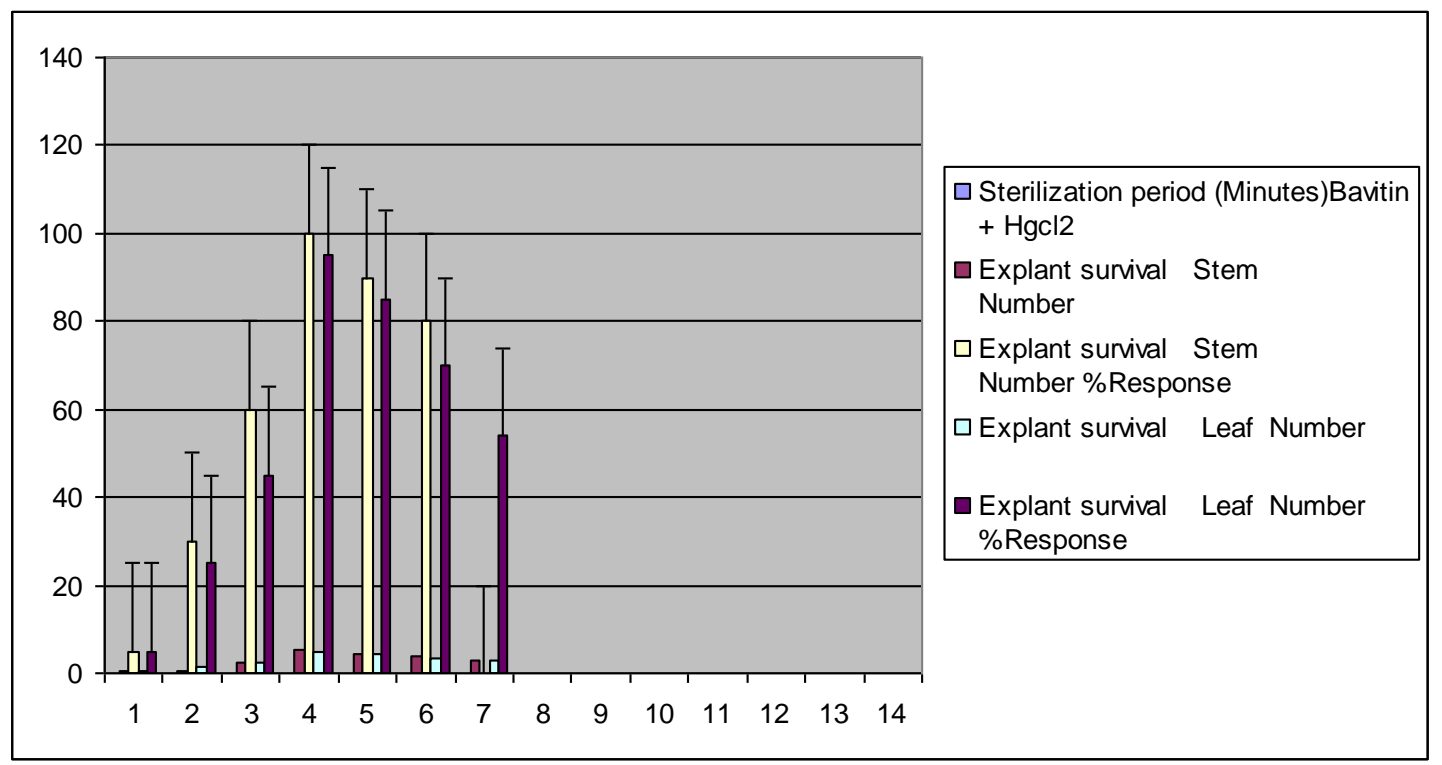

Table 4 - Screening of leaf size and stem length for root induction on 20th day of incubation

\begin{tabular}{|c|c|c|c|c|}
\hline Treatment & Leaf size & Response & $\begin{array}{l}\text { Stem } \\
\text { length }\end{array}$ & Response \\
\hline T1 & 0.25 & $0 \%$ & 0.5 & $0 \%$ \\
\hline T2 & 0.5 & $30 \%$ & 0.75 & $26 \%$ \\
\hline T3 & 0.75 & $58 \%$ & 1 & $48 \%$ \\
\hline T4 & 1 & $82 \%$ & 1.25 & $90 \%$ \\
\hline T5 & 1.25 & $100 \%$ & 1.5 & $100 \%$ \\
\hline
\end{tabular}


Screening of leaf size and stem length for root induction on $20^{\text {th }}$ day of incubation

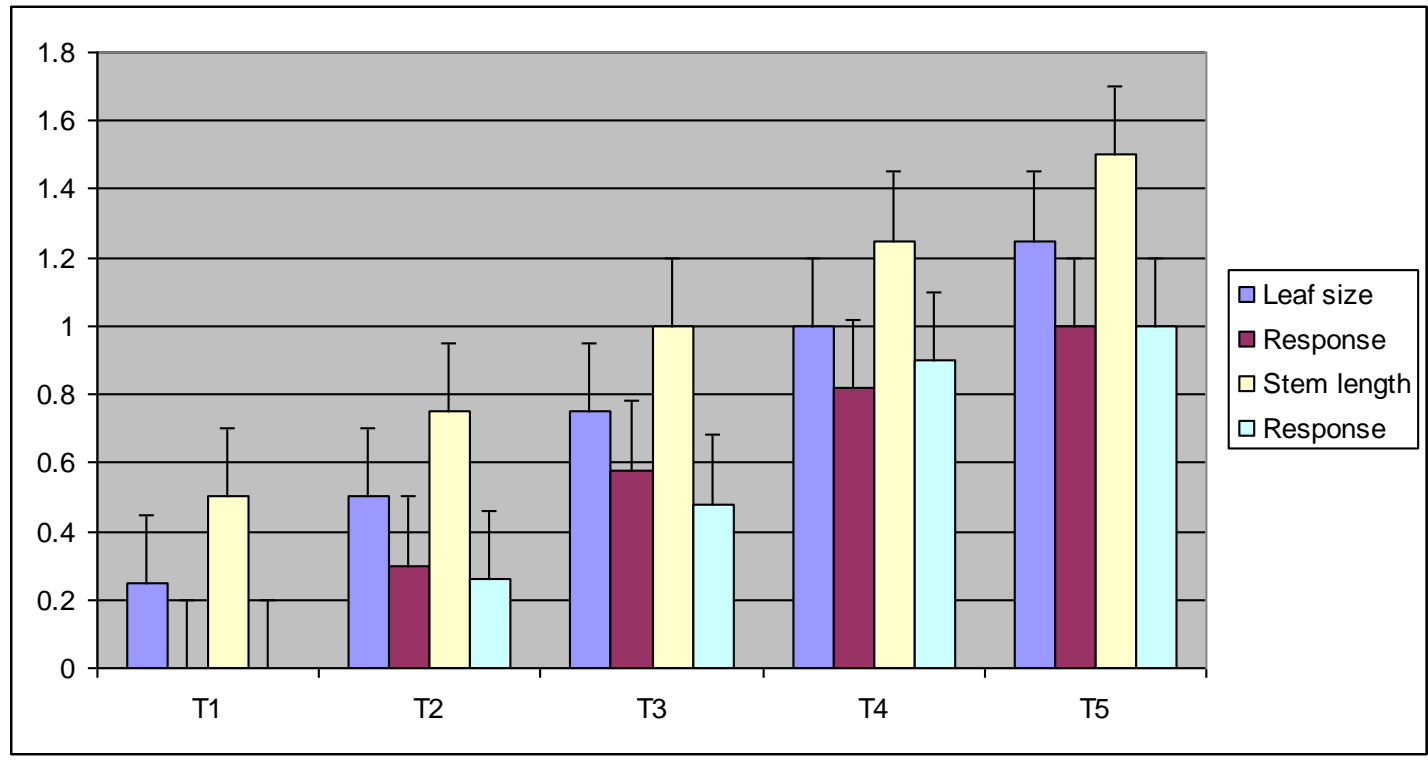

Table 4 - Average Root Length Obtained from the treatment

\begin{tabular}{|l|r|r|r|r|}
\hline Treatment & Leaf size & $\begin{array}{l}\text { Length } \\
\text { (cms) }\end{array}$ & Stem size & Length(cms) \\
\hline T1 & 0.25 & 0 & 0.5 & 0 \\
\hline T2 & 0.5 & 0.31 & 0.75 & 0.25 \\
\hline T3 & 0.75 & 1.32 & 1 & 1.01 \\
\hline T4 & 1 & 2.72 & 1.25 & 1.23 \\
\hline T5 & 1.25 & 3.98 & 1.5 & 2.98 \\
\hline
\end{tabular}

Average Root Length Obtained from the treatment

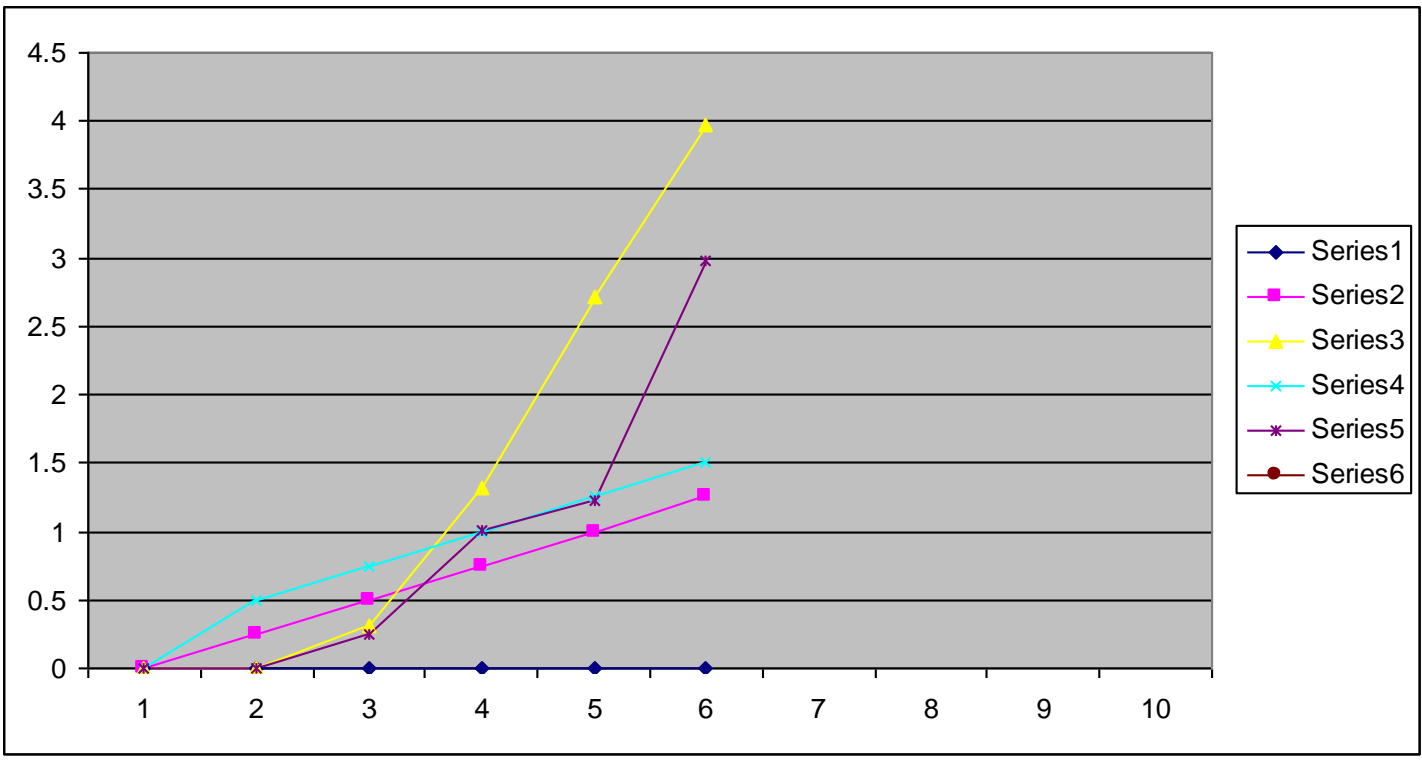

